\title{
The Practice of Affective Teaching: A View from Brain Science
}

\author{
Wenhai Zhang \& Jiamei Lu \\ Shanghai Normal University \\ Shanghai 200234, China \\ Yancheng Institute of Technology \\ Yancheng 224002, China \\ E-mail: zwh2007106@126.com
}

\begin{abstract}
In the educational field, the cognitive side of learning usually gets a great deal of attention, but affective factors are always ignored. Recently neuroscience researches have accumulated much knowledge about the relationship between cognition and emotion, which attract educators' concern. This article aims to glean brain science knowledge about emotions, further recognize the functions of emotions, and relate these to affective teaching to effectively improve students' learning. The author argues that cognition and emotion deeply interact for overlapping cognitive and emotional brain areas which is quite malleable and influenced by maturation and experience, and that emotions possess motivational, informative, regulative, protective functions, and that learning relies on emotions state, which determines what we pay attention to and what we learn. In conclusion, teachers first eradicate threats, and then involve emotions into students' learning through affective teaching including modeling emotion exhibition about the learning and the subject, affective processing material, making affective instruction. The future educationists need to strengthen contact with other scientists such as neuroscientists, psychologists, socialists, biologists, to work hard together to shrink the gap between education and neuroscience.
\end{abstract}

Keywords: Brain, Education, Neuroscience, Emotion

Since Plato (360 B.C.), a tradition in western thought contrasts the animal, reflective body with the uniquely human, relational mind or soul. This philosophical dualism has had a profound impact on meta-psychological theories of emotion and the mind. Emotions is either ignored by psychologists and neuroscientists or treated merely as an unwelcome source of noise or bias in relationship to "normal" cognitive function. In the same way, in the educational field, the cognitive side of learning usually gets a great deal of attention, but the domain of emotions, the so-called affective side of learning has long been neglected. In fact, the affective aspect of learning is the key interaction between how students feel, act, and think. There is no separation of mind and emotions; emotions, thinking, and learning are closely linked together. When we ignore the emotional components of any subject we teach, we actually deprive students of meaningfulness (Caine \& Caine, 1991).

Luckily, neuroscience has begun to uncover the biological basis of learning and emotions with many non-invasive technologies such as fMRI, ERP, MEG during the past decades. Using the newly-built knowledge about the brain from the neuroscience, educationists can reconsider the relationship between emotions and cognition, gain a further insight into learning process, reconstitute the role of emotions in teaching and learning so that teachers take full use of affective factors in instructional process to maximize students' learning. This article is divided into three parts: (1) to depict the emotional brain; (2) to recognize the functions of emotions; (3) to take the practice of affective teaching.

Before proceeding, it is worth considering some definitions. The term "affect" is very broad, and has been used to cover a wide variety of experiences such as emotions, moods, and preferences. In contrast, the term "emotion" tends to be used to refer to fairly brief but intense experiences, although it is also used in a broader sense. Finally, "mood" or "state" is term describing low-intensity but more prolonged experiences.

\section{Emotions and Brain}

\subsection{The brain structure involving emotions}

The limbic system. Inspired by Papez's analysis, Mclean (1993) proposed that the human fore brain includes three distinct system, each of which developed in a distinct phase of vertebrate evolution. Apart from the hypothalamus, the earliest and most basic part of forebrain is called the striated region. The area became enlarged with the evolution of 
reptiles. By contrast to reptiles, every mammal is born in close association with another, and broadly speaking mammal are social creatures. As mammals diverged from reptiles in the course of evolution, the limbic system developed to enable mammals' increasing sociality. The third part is the neocortex, which is the highest and most recently evolved level.

The amygdale: appraisal of fear or negative emotions. Joseph LeDoux (2000) has argued that the limbic system theory of emotion offered by Maclean (1993) is a vague and flawed and inadequate theory of the evolutional brain. He argued that the amygdala is the central emotional computer for the brain: it is the appraisal mechanism for emotions. The amygdala is a heterogeneous structure that, in primates, consists of at least 13 anatomically and functionally distinct subnuclei (Amaral et al, 1992; LeDoux, 2000). Besides the complex internal structure, the amygdala has extensive external anantomical connections, which allow the amygdala to integrate sensory input from all modalities and to affect autonomic and motor output systems.

As well as inputs from the visual and auditory cortex, the amygdala receives visual and auditory inputs directly by way of the thalamus before these reach the associative cortex (LeDoux, 1996). The direct thalamic pathway to the amygdala is shorter and thus faster. However, the thalami-cortico-amygdala pathway, is longer and slower. The thalamic pathway is sufficient for the rapid triggering of emotion by simple stimulus features, whereas the cortical pathway appears to be needed for emotional reactions coupled to perceptually complex stimulus objects (LeDoux, 1995).

The nucleus accumbens: central to positive emotions. The nucleus accumbens processes afferent input from many of the cognitive and limbic areas of the brain, including the prefrontal cortex (PFC), hippocampus (HC), amygdala, and thalamus. The output neurons of the nucleus accumbens send axon projections to the ventral analog of the globus pallidus, which, in turn, projects to the mediodorsal (MD) nucleus of the dorsal thalamus, which projects to the prefrontal cortex. Other efferents from the nucleus accumbens include connections with the substantia nigra and pontine reticular formation.

In the 1950s, Olds and Milner implanted electrodes into the septal area of the rat and found that the rat chose to press a lever which stimulated it. It continued to prefer this even over stopping to eat or drink, which suggests that the area is the "pleasure center" of the brain.

The insula: disgust recognition. The insular cortex is a multimodal sensory region with visceral, gustatory, somatosensory, visual, and auditory afferents and reciprocal connections to amygdala, hypothalamus, cingulate gyrus, and OFC. In addition to its role in interoceptive representation and autonomic control, the insula has also been implicated in the acquisition of inhibitory avoidance behavior.

Although the insula may exhibit functional specialization for disgust, its role in emotional processing is not restricted to one particular emotion. Critchley et al. (2002) have suggested that insula may play a crucial role in mediating the influence of peripheral autonomic arousal on consciously experienced emotional states,a suggestion that would accord with a role for this region in subjective aspects of emotion(i.e., "feeling" states)(Frackowiak et al., 2004).

The anterior cingulated cortex: emotional monitor. The anterior cingulate cortex (ACC) can be divided anatomically based on attributed functions into executive (anterior), evaluative (posterior), cognitive (dorsal), and emotional (ventral) components. An affective ventral subdivision of ACC (comprised of Brodmann's area 25, 32, 33) has connections to amygdala, nucleus accumbens, orbitofrontal cortex, anterior insula, and autonomic brain stem regions. A dorsal cognitive subdivision (Brodmann's caudal area 24 and 32 and cingulate motor area) has anatomical connections with parietal cortex, posterior cingulate, supplementary motor area, and dosallateral prefrontal cotex.

The ACC is connected with the prefrontal cortex and parietal cortex as well as the motor system and the frontal eye fields (Posner \& DiGirolamo, 1998) making it a central station for processing top-down and bottom-up stimuli and assigning appropriate control to other areas in the brain. The ACC seems to be especially involved when effort is needed to carry out a task such as in early learning and problem solving. Many studies attribute functions such as error detection, anticipation of tasks, motivation, and modulation of emotional responses to the ACC. Relatively, one of ventral anterior cingulate may be to monitor and evaluate external stimuli (especially when aversive or painful) and select appropriate responses with respect to ongoing emotional priorities and goals (Davidson et al., 2002).

The prefrontal cortex: emotional regulation. The prefrontal cortex (PFC) can be divided in several ways, one of which is into three basic areas: The (OFC) and ventromedial areas (vm-PFC); the dorsolateral prefrontal cortex (dl-PFC); the anterior and ventral cingulate cortex. Other areas that can be distinguished are the ventrolateral cortex (vl-PFC), the medial prefrontal cortex (m-PFC), and the rostral prefrontal cortex (r-PFC).

Although the PFC is usually deemed to be the site of higher cognitive control, it has also been linked to affective processing and regarded as centers of regulation or executive control (Gazzaniga et al., 2002) because regions of prefrontal cortex have close connections with the limbic system, including dense reciprocal connections to the amygdala and the nucleus accumbens. This means the PFC play an important role in the regulation of emotion. Emotion regulation involves many neural structures, that include several regions of the PFC, the amygdala, hippocampus, 
hypothalamus, ACC, insular cortex, ventral striatum, both top-down and bottom-up processes.

\section{Plasticity}

Contemporary researches agree that human development involves a dynamic interplay of nature and nurture (OECD, 2007). Nature and nurture work hand in hand. The brain is malleable, not only being influenced by inheritable genes but also by the physical, social, and cultural environments, which set a basis for education.

Synaptic plasticity in the amygdale. Learning is done through the alternation of synaptic efficacy at the cellular level. Long term potentiation (LTP) has tentatively accepted as essential to the actual physical process of learning. The classic form of LTP is dependent upon glutamatergic transmission, and specifically upon NMDA receptor function, and has been widely discussed as a possible element in the physiology of learning and memory. Studies in a number of systems have implicated excitory glutamatergic transmission and NMDA receptor function in memory formation. NMDA receptor function is a mechanistic link between LTP and the plasticity underlying fear conditioning. In thalamo-amygdala pathway, glutamate is present in presynaptic neurons and in the post synaptic terminal region (LeDoux, 1995). LTP has been produced in pathways to the amygdala originating in the auditory thalamus, neocortex and hippocampus.

Development. Neuroscience has shown the surprising extent to which the brain is still development during childhood and adolescence and even throughout adult life in white matter (WM) volumes (Giedd et al., 1999), which reflects an increase in associative cognitive activity as distributed brain modules become more and more integrated by synaptic pruning (in which infrequently used connections are eliminated) and myelination. Unlike WM increases during childhood and adolescence, the gray matter (GM) trajectories follow an inverted U-shaped path. Age of peak size for GM volumes differs, varies by region, and is generally earlier in females than in males. The age of peak GM density is earliest in primary sensorimotor areas and latest in higher order association areas that integrate those primary functions such as the dorsolateral prefrontal cortex, inferior parietal, and superior temporal gyrus. Part of the GM changes may be related to synaptic proliferation and pruning (Huttenlocher, 1994).

There is a changing balance between competing neuronal networks as different cognitive and emotional systems mature at different rates. FMRI consistently shows an increasing proportion of frontal versus striatal or limbic activity from childhood to adulthood for a variety of cognitive tasks (Rubia et al., 2006). Some changes in limbic reward and motivational systems seem to be associated with the onset puberty, whereas other changes occur earlier or well after the advent of puberty. For example, in an fMRI study of 37 subjects aged 7-29 years that assessed response to rewards, adolescent nucleus accumbens response was equivalent to that in adults, but adolescent orbitofrontal activity was similar to that in children

All these overt changes in the brain connectivity help make childhood and adolescence a good time to learn in cognitive, affective, social fields.

Experience. The physical structure of the brain changes in part as the result of experience. The brain is made up of networks of interconnecting nerve cells. Experience gradually modifies the connections between neurons following a "use it or lose it" rule (Hinton, Miyamoto, \& Della-Chiesa, 2008). Researches indicate that hippocampus continues plasticity in adult. This means that the hippocampus is designed for lifelong learning and adaptation to new situations and experience, and adaptation can even bring about changes in its structure. Researches relating rich environment indicate that the adult rat brains form new synapses in response to new experience (Greenough, West, \& DeVoogd, 1978). Joffe (1997) found that primates who live in complex social systems tend to have a relatively log childhood and a relatively large neocortex. One of the primary functions of childhood is provided the experiences needed to adapt the relatively open or plastic brain systems that support social competencies to the local social ecology.

When the brain has been rewired by experience, lives are changed. Education is about shaping the brain's functional organization. Learning to read, to use calculus, or to dance all draws upon diverse neurological capacities, and instruction reshapes not only behavior on these tasks but also functional organization of the utilized portions of our brains.

\section{The Functions of Emotions}

Evolutionarily, although emotions are shared by human beings and animals, emotions have completely different meanings for mankind not only because in complex social environments but also human beings have higher cognitive capabilities than other primates. Advocating the bio-psycho-social positions, we hold that emotions own the following functions for learning and life.

\subsection{Motivational}

Emotion and motivation are closely linked. Emotions can elicit motivation, which moves people to behave, think, and feel the way they do, and prepare us for action and thought. For example, in an emergency when we see an angry dog charging toward us, the Sympathetic Nervous System immediately causes an increase in blood pressure, a faster heart 
rate, more rapid breathing for greater oxygen intake, and more efficient blood flow to the brain and major muscle groups. All of these changes prepare us for action. By contrast, the parasympathetic nervous system (PNS) calms the body. When the PNS is activated, heart rate and blood pressure drop, stomach activity and food digestion increase, and breathing slow down. Emotional motivation tends to take place on a widespread and unconscious level, but can also happen consciously. For instance, in situations where students seem ambivalent or conflicted, take a look at the possibility that there are neural representations of both positive and negative outcomes. If they can identify the negative outcome that is feared, they can apply a corrective that frees the individual to move forward toward the positive.

\subsection{Informative}

Emotions are a crucial source of information for learning and daily decision-making. Although extremes of emotion are generally harmful to our best thinking, a middle degree and appropriate emotions speed up decision-making enormously. Schwarz and Clore (1983) proposed the perspective of the feelings as information. When making evaluative judgments, individuals often do not use presumably effortful analytic judgment strategies such as recalling the various positive and negative attributes of the object and then forming a summary judgment but rely on a simplifying judgment method called the "feeling heuristic".

From the classic case of Phineas Gage, we can see how damage to his OFC altered his emotions and ability to live a moral life. Modern neuro-psychological studies of patients with OFC lesions suggest a psychological disorder of emotional disinhibition. During an emotional event, the OFC would normally signal to the organism information about the consequences (pleasant/unpleasant) of one's actions.

Without emotions students would be impossible to make a proper moral judgment and to live a normal social life. They need emotions to provide information to understand their own body-mind states, guide and maintain their own attention in learning process, regulate their goals to enhance learning and communicative abilities, and redirect their behaviors.

\subsection{Regulative}

Emotions deeply interact with cognitive processes. Modern functional neuroimaging studies have indicated that the anatomical basis of emotions and cognitive processing have considerable overlap. For example, Sad mood, in both healthy participants and those with clinical depression, has been shown to influence the activity of a common set of prefrontal and limbic brain regions like the amygdale (Davidson et al., 2002; Mayberg et al., 1999). Many of these are also implicated in cognitive functions. The overlap between the brain areas modulated by sad mood and cognition is consistent with a shared, interactive neural architecture for cognition and mood.

Emotions can regulate cognitive activity and guide cognitive processes like perception, attention, memory, and so on. Bower (1981) and Gilligan and Bower (1984) put forward a semantic network theory, which proposed that emotions are units or nodes in a semantic network, with numerous connections to related ideas, to physiological systems, to events, and to muscular and expressive patterns. Mood congruity can affect perception, retrieval as well as learning, which depends on mood state. Current feelings tend to bias our recall of past emotions. Consistent with the thesis of emotion-congruence, when in a happy mood, participants were quicker at identifying happy than sad words. When sad, they were quicker at identifying sad than happy words. Both real life and in the laboratory, emotionally salient material is remembered better than neutral material.

The abilities of emotional regulation in childhood and adolescence are co-influenced by biological maturation and experiences. Because their PFC matures later than other emotional structures, many students are subjective to emotional bewilderments. But their brains are comparatively malleable, teacher can help them learn many strategies to deal with emotional problems, accumulate experiences about emotional regulation and take advantage of emotions to improve learning.

\subsection{Protective}

Generally speaking, from a long-term view positive emotions can improve health; In contrast, negative emotions can harm health. Differences in underlying neurological activation for trait positive affect (FA) and negative affect (NA) suggest that there are fundamental differences in how the brain represents these traits. These differences may similarly imply differences in the direction and manner that they influence health. For example, PA-associated activations occur primarily in the left frontal cortex, whereas NA occurs in the right frontal cortex (Davidson, Jackson, \& Kalin, 2000). Incidentally, left prefrontal cortex (PA-like) activation is also associated with improved immune function (Davidson et al., 2003; Kang et al., 1991). Neurotransmitters may also respond differently to PA than to NA. For example, trait PA was associated with increased serotonergic function after controlling for NA (Flory et al., 2004).

Fredrickson (1998) has proposed a new model of the function of positive emotions. According to the broaden-and-build model, positive emotions broaden people's "momentary thought-action repertoires" and build their physical, social, and intellectual resources. Positive emotions produce more creative and flexible thought, help students in forming important associations and exploring the environment, and also aid them building interpersonal resources by motivating 
them to approach others, to cooperate, to express affection, and to build bonds.

The above four functions interplay in many conditions and are difficult to be clearly differentiated. What's important, teachers can involve emotions into students' learning and make full use of these emotional functions to establish preponderant environments and design instruction process to change the weakness in traditional teaching process where emotions are neglected and excluded from. In succession, we mainly discuss the practice of affective teaching about how to apply emotions and affection into classroom and instruction.

\section{The Practice of Affective Teaching}

From an evolutionary perspective, human being brain is biologically designed to allow the individual to survive reproduce in the social, biological, and physical environments. As a basic function of the brain, Emotions are a form of learning. We have learned what to love, when and how to care, whom to trust, the loss of esteem, the joy of discovery, and the fear of failure. So, affective teaching firstly aims to develop students' affective quality. Secondly, the brain areas involving emotion and cognition highly overlap. This means more than that they just influence each other. None of the ingredients that we deal with in education, such as "concept" and "emotions" and "behaviors," is separate. They influence and shape each other (Caine \& Caine, 1991). Affective teaching ought to play a key role of emotions to optimize cognition and enhance students' integrative quality.

\subsection{Eradication of threats}

We must first eradicate a variety of threats from the environment and build a secure learning environment to maximize learning achievement before we involve emotions into students' learning. Threats activate defense mechanisms and behaviors that are good for survival but bad for learning because survival always overtops complex problem solving. For example, when faced fear and danger, the brain jumps into high gear. The amygdala can intercept information from the sensory channels through shortcut circuit - the thalamo-amygdala pathway. This immediately generates unconscious processing, interrupting the ongoing learning activity, occupying limited brain processing resources. These usually lead learning to fail. Students are unable to create connections, make meaning and to gain higher levels of knowledge structure.

In stressful environments, the hypothalamic-pituitary-adrenal axis activates and releases a peptide called cortisol. It elicits a serial physical reactions including inhibition of the immune system, increasing blood pressure, straining the large muscles. These can temporarily help individual cope with stress. But chronically high cortisol level leads to the death brain cells in the hippocampus (Vincent, 1990), a relay site involving information storage and retrieval. Chronic stress also impairs a student's ability to sort out what's important and what's not. A stressful physical environment is linked to student failure. Learners with lower stress can put together relationship, understand broad underlying theories, and integrate a wider range of material. Crowed conditions, poor peer relationships, disgust student-teacher relationships can matter.

Fortunately, there are many approaches to reduce stress for students. One is to encourage students to participate gregarious activities in respective ways. Drama, peer support, games, exercise, discussions, and celebrations can help students release stresses. Through kind humor and good natured classroom interactions that honor students and opportunities to see "failure" as feedback for progress and growth, teacher can go a long way toward aiding children to replace the pattern of fear with confidence and eager anticipation. Anyway, never tolerate students bullying one another in classroom. Another is to fulfill intrapersonal needs. Advocate students to keep daily writing, make creative writing. These provide students chances to reflect personal experience and reorganize self. Other strategies include: to avoid finger-pointing, scolding, and detention; ensure learners to have enough rest time; direct students learn to be responsible for one's own behaviors; teach students stress management, and the like.

To sum up, for most learning conditions, low to moderate levels of stress are best. High stressor threat has no place in schools. Stress, threat, and learned helplessness must be removed from learning environments.

\subsection{Involvement of emotions in teaching and learning}

With the increasing understanding of the relationship between emotion and cognition, people realize that emotions in many contexts are rational in that they help individuals respond adaptively to the environment. Now we must throw away the wrong idea of regarding emotions as always irrational or having nothing to do with the ways we think. Emotions prioritize to be processed and set up mental states which provide the context for learning activities and guide cognitive processes in rational, adaptive fashion. In addition, we remember that which is most emotional laden. Learning relies on emotions state, which determines what we pay attention to and what we learn. So learning cannot be separated from emotions. Emotions are essential to learning. Consequently, teachers cannot ignore emotion as a vital influence in the learning process. As educators, we must involve emotions into instruction to maximize students' learning. Teachers who help their students feel good about learning through classroom success, friendships, and celebrations are doing the very things the student brains carve. 
From static analysis, there are three basic affective resources in instruction: teacher, text, and students. The three factors interact and make up instructional affective field, which provide possibility for affective instruction.

Modeling. As a basic affective source, teacher not only possesses rich affective experiences, but also receives outside stimulus and exerts the influence on students. At the same time, as instructional organizers and directors, teachers' leading status determines them more impact. No doubt, teachers have more experiences and more mature in the affective field than students. They have enough capacities to use many functions of emotions to optimize instruction. First, teachers can and must model excellent emotion exhibition. They ought to demonstrate the love learning, and show sincere enthusiasm about the subject they teach inside and outside classroom. They must regulate their own emotion state, shape good mood, and bring positive emotions into classroom. Second, teachers should grasp students' individual desires, dreams, expectation to lead to personal goal setting, care for them, respect their personal strength, and help them discover a passion for learning and diminish their weakness.

Affective processing of material. Text includes a great deal of affective factors. Generally, we classify text into three categories: salient, connotative, and inexistent. Teachers can take different strategies for different texts. Salient text means that affective factors in text can be felt directly, including song, dance, picture, sculpture, and video, and the like. Teachers can "demonstrate" affects directly through multimedia or many field activities. Connotative text mainly reflects objective facts and has not very obvious affective factors, but reader can unconsciously feel some affects in these facts. Teachers can "dig" these affective factors in the text with affection transferring. Although affective cues seem not to exist in some texts like mathematics, teachers can "color" the text by relating the text with students' affective experiences.

Affective instructional process. Affective instructional process includes four basic stages: elicitation, edification, inspiration, and regulation. At the first stage, teachers ought to elicit students' interests, and mobilize students to participate learning. Teachers need to grasp students' needs and match these needs with teaching contents so that instructional materials become Students' learning Inducement. Thus, teachers help students change study attitude from negative to positive. Next, teachers take advantage of affective field that is built at the first stage to edify students. Teachers and students immerse their brains into texts. Now students may experience, express, recognize, regulate their and others' affects safely and comfortably, so they enhance their affective abilities (or emotion intelligence). With the increasing of task difficulty, students gradually lose part drive. Teachers must inspire students by encouraging evaluation to help them get a sense of success. Students can get more positive emotions from success. These positive experiences help students make meaning by relating current learning to the past and the future. As classroom managers, teachers regulate students' mental state to ensure students' leading mood to be happy and interesting all the while. Obviously, teachers can flexibly regulate instructional processes but not fix sequent stage as above.

\section{Conclusion}

In all, affective teaching can make full use of emotional functions gained from neuroscience knowledge and improve students' classroom learning. Although there is still a gap between affective education and brain science, researches about their relationship will gradually attract the attention of many scientists and teachers and become the mainstream. Because researches concerning emotions relate to many subjects, the future educationists need to strengthen contact with other scientists such as neuroscientists, psychologists, socialists, biologists, to work hard together to deeply understand the relationship among brain, cognition, emotion, and to commit themselves to apply the knowledge about the brain researches into instructional practice to form co-prosperity of cognition and affection.

\section{Acknowledgement}

This research is supported by The Philosophy and Social Science Project Fund of Colleges and Universities in Jiangsu Province of China in 2008, and the number is 08SJD7100068.

\section{References}

Amaral, D. G., Price, J. L., Pikanen, A., and Carmichael, S.T. (1992). Anatomical organization of the primate amygdaloid complex. In J. P. Aggleton(Ed.), The Amygdala: Neurobiological Aspects of Emotion, memory and Mental Dysfunction (pp.1-66). New York: Wiley-Liss.

Bower, G.H. (1981). Mood and memory. American Psychologist, 36, 129-148.

Caine, R.N., Caine, G. (1991).Making connections: teaching and the human brain. Alexandria, Virginia: Association for Supervision and Curriculum Development.

Davidson, R.J., Kabat-Zinn, J., Schumacher, J., Rosenkranz, M., Muller, D., Santorelli, S. F., et al. (2003). Alternations in brain and immune function produced by mindfulness meditation. Psychosomatic medicine, 65, 564-570.

Davidson, R. J., Pizzagalli, D., Nitschke, J. B., \& Putnam, K. (2002). Depression: Perspectives from affective neuroscience. Annual Review of Psychology, 53, 545-574. 
Davidson, R.J., Jackson, D.C., \& Kalin, N. H. (2000). Emotion, plasticity, context, and regulation: Perspectives from affective neuroscience. Psychological Bulletin, 126, 890-909.

Flory, J. D., Manuck, S. B., Matthews, K. A., \& Muldoon, M. F. (2004). Serotonergic function in the central nervous system is associated with daily ratings of positive mood. Psychiatry Research, 129(1), 11-19.

Fredrickson, B. L. (1998). What good are positive emotions? Review of General psychology, 2, 300-319.

Gazzaniga, M.S., Ivry, R.B., and Mangun, G.R. (2002). Cognitive neuroscience. New York: Norton.

Giedd, J.N., Blumenthal, J., Jeffries, N.O. et al. (1999). Brain development during childhood and adolescence: a longitudinal MRI study. Nat Neurosci, 2, 861-863.

Gilligan, S.G., \& Bower, G.H. (1984). Cognitive consequences of emotional arousal. In C. Izard, J. Kagen, \& R. Zajonc (Eds.), Emotions, cognition, and behaviour. New York: Cambridge University Press.

Greenough, W.T., West, R.W, \& DeVoogd, T.J. (1978). Subsynaptic plate perforations: changes with age and experience in the rat. Science, 202, 1096-1098.

Hinton, C., Miyamoto, K. \& Della-Chiesa, B. (2008). Brain research, learning and emotions: implications for education research, policy and practice. European Journal of Education, 43(1), 87-103.

Huttenlocher, P.R. (1994). Synaptogenesis in human cerebral cortex. In Dawson G, Fischer K (Ed.). Human Behavior and the Developing Brain. New York: Guild Press.

Joffe,T.H. (1997). Social pressures have selected for an extended juvenile period in primates. Journal of Human Evolution, 32, 593-605.

Kang, D. H., Davidson, R. J., Coe, C. L., Wheeler, R. E., Tomarken, A. J., \& Ershler, W. B. (1991). Frontal brain asymmetry and immune function. Behavioral Neuroscience, 105, 860-869.

LeDoux, J. E. (1995). Emotion: Clues from the Brain. Annu.Rev.psycho, 46, 209-235.

LeDoux, J. E. (1996). The Emotional Brain. New York: Simon \& Schuster.

LeDoux, J.E. Emotion Circuits in the Brain. Annu.Rev psycho.2000, 23:209-235.

Mayberg, H. S. et al. (1999). Reciprocal limbic-cortical function and negative mood: Converging PET findings in depression and normal sadness. American Journal of Psychiatry, 156, 675-682.

Mclean, P. (1993) Cerebral evolution of emotion. In Lewis, M. \& Haviland, J.M. (Eds.), handbook of emotions (pp.67-83). New York: Guilford.

OECD. (2007). Understanding the Brain: The Birth of a Learning Science. Paris: OCED Publishing.

Olds, J., Milner, P. (1954). Positive reinforcement produced by electrical stimulation of septal area and other regions of rat brain. Journal of Compared Physiological Psychology, 47 (6), 419-427.

Posner, M.I., \& DiGirolamo, G.J. (1998). Executive attention: Conflict, target detection, and cognitive control. In R. Parasuraman (Ed.), The Attentive Brain: MIT Press.

Rubia, K., Smith, A.B., Woolley, J. et al. (2006). Progressive increase of frontostriatal brain activation from childhood to adulthood during eventrelated tasks of cognitive control. Hum Brain Mapp, 27, 973-993.

Schwarz, N., \& Clore, G.L. (1983). Mood, misattributions, and judgments of well being: Informative and directive functions of affective states. Journal of Personality and Social Psychology, 45, 513- 523.

Vincent, J.D. (1990). The Biology of Emotions. Cambridge: Basil Blackwell. 